Pensamiento Crítico N. ${ }^{\circ}$ 9, pp. 139-149

\title{
El marco teórico de los errores y vacíos de la Teoría Monetaria Clásica y Keyne- siana sobre el impacto de la expansión monetaria en las tasas de interés y los ciclos económicos (caso peruano 2000-2007)
}

Econ. Pablo Rivas Santos

\section{RESUMEN}

El presente artículo trata sobre los errores y vacíos de la Teoría Keynesiana y Clásica sobre la neutralidad del dinero. Si los economistas clásicos y keynesianos no se hubieran equivocado en materias referentes a los problemas monetarios, aferrándose después a sus errores, difícilmente podrían prevalecer todas esas nefastas políticas, inspiradas en populares teorías monetarias, que han desorganizado la política monetaria en casi todos los países.

Palabras clave: Dinero neutral, poder adquisitivo del dinero, políticas contracíclicas, inflación. 


\title{
Pablo Rivas Santos
}

\begin{abstract}
The present article deals about errors vacuum in classical Keynesian theory on the neutrality of money. If classical and Keynesian economists have not been wrong in matters relating to monetary problems, after clinging to his mistakes, could hardly prevail all these disastrous policies, inspired by popular monetary theories, which have disrupted monetary policy in almost all countries.
\end{abstract}

Keywords: Money neutral purchasing power of money, counter-policies, inflation.

\section{INTRODUCCIÓN}

La formulación de la Ciencia Económica dependió de la posibilidad del cálculo económico; hasta tal punto que los economistas clásicos y keynesianos no se dieron cuenta de los decisivos problemas que implicaba el cálculo económico. El cálculo económico es categoría inherente a la acción humana practicada bajo específicas condiciones. El cambio interpersonal y el intercambio de mercado, basado en el uso del dinero y los precios, son fenómenos típicos y exclusivos de cierta organización económica de la sociedad. El cálculo económico solo es posible a través de los precios monetarios. Los escritos de los economistas clásicos y keynesianos adolecen de esas imperfecciones generadas por su errónea visión del cálculo económico.

La teoría económica permite darnos cuenta cómo la personal elección de cada individuo estructura los precios de mercado en el mundo del cambio interpersonal. La teoría económica - en ciertos aspectos- no es del todo satisfactoria y, además, un léxico imperfecto viene a veces a desfigurar su contenido. En esencia, la teoría económica es irrefutable. La labor de completarla y mejorarla (en aquellos aspectos que necesita enmienda) debe consistir en lógica reestructuración del pensamiento de sus autores.

Para reducir los complejos fenómenos de mercado a la universal y simple categoría de preferir "a" en vez de "b", la teoría económica recurre a construcciones imaginarias (Modelos). Los modelos, sin correspondencia con el mundo real, son indispensables herramientas del pensar. Una de las cuestiones de mayor trascendencia científica consiste en saber eludir los errores en que se puede caer; cuando los modelos se manejan de modo imprudente. 


\section{El marco teórico de los errores y vacíos de la Teoría Monetaria Clásica y Keynesiana}

La primera teoría económica recurrió al planteamiento que supone la existencia de un mercado en que sólo habría cambio directo. En tal planteamiento, el dinero no existe; unos bienes y servicios se cambian por otros bienes y servicios. Sin embargo, tal modelo es inevitable, ya que para darnos cuenta de que son cosas de primer orden las que se cambian por otras cosas de primer orden, conviene excluir el dinero del análisis (-instrumento del cambio interpersonal- con su función intermediaria). Sin embargo, es necesario cuidarse de errores en que se puede caer al manejar tal modelo.

\section{EL ROL QUE DESEMPEÑA EL CAMBIO DIRECTO EN LA TEORÍA MONETARIA}

Grave error provocado por errónea interpretación de ese modelo, fue suponer que el dinero es neutral. Con arreglo a tal supuesto, lo que diferencia el cambio directo del cambio indirecto consiste en el uso del dinero. La interpolación de la moneda en la transacción no afectaba las bases fundamentales de la operación. Se pensaba que las profundas fluctuaciones del poder adquisitivo del dinero que provocaron graves convulsiones en todo el sistema de intercambios eran supuestos excepcionales, provocados por medidas inoportunas; solo la moneda mala podía dar lugar a similares desarreglos. Ello suponía caer en error, al abordar las causas y efectos de esas fluctuaciones. Se creía que las fluctuaciones del poder adquisitivo del dinero afectaban, por igual y al mismo tiempo, a los precios; la ficción de la neutralidad económica del dinero vierte tal conclusión. Se llegó a suponer que cabía estructurar la teoría económica sobre el cambio directo. Una vez logrado esto, bastaría (para completar el sistema) con insertar los conceptos dinerarios en los correspondientes teoremas. A tal dineraria complementación, se daba poca importancia, ya que parecía que no modificaría sustancialmente ninguno de los conceptos fundamentales; y, la misión esencial de la economía consistía en analizar el cambio directo. Aparte de tal examen, lo más que podía interesar era el estudio de los problemas suscitados por la moneda mala.

Los economistas (basados en tales supuestos) se desentendían del cambio indirecto, abordando superficialmente los problemas monetarios; que consideraban simple apéndice escasamente relacionado con sus estudios básicos. Las cuestiones del cambio indirecto quedaron relegadas a segundo plano ${ }^{1}$. Sin embargo, con el paso del tiempo

1 Influencias de índole política contribuyeron a que se descuidara el examen de los problemas del cambio indirecto. la gente no quería abandonar aquellas tesis según las cuales las crisis son mal típico del sistema 


\section{Pablo Rivas Santos}

los economistas se dieron cuenta, que algunos de los más trascendentales y difíciles problemas de la teoría económica surgían en la esfera del cambio indirecto, resultando incompleta toda teoría económica que descuidara dicha materia. El que los economistas comenzaran a preocuparse por temas como la proporcionalidad entre tasa de interés real y tasa de interés nominal; el que se concediera cada vez mayor importancia a la teoría monetaria de los ciclos económicos y el que se rechazaran las teorías que suponían la simultaneidad y uniformidad de las fluctuaciones del poder adquisitiva del dinero, evidenciaba que había aparecido una nueva tendencia en el pensamiento económico.

Más perjudicial fue un segundo error, provocado por el poco riguroso manejo de ese modelo que se limita a contemplar un mercado que solo conoce el cambio directo.

Grave error era suponer que los bienes o servicios objeto de intercambio deberían tener entre sí el mismo valor. Se consideraba el valor como una cualidad objetiva, intrínseca, inherente a las cosas, sin advertir que el valor es el reflejo del ansia con que el individuo aspira al bien que le apetece. Se suponía que mediante el acto de medir, la gente establecía el valor de los bienes y servicios, procediendo luego a intercambiarlos por otros bienes y servicios de igual valor. Por el contrario, la Economía moderna se basa en el conocimiento de que el cambio directo surge a causa del diferente valor atribuido por las partes a los objetos intercambiados. La gente compra y vende, única y exclusivamente, por cuanto valoran en menos lo que dan que lo que reciben. De ahí que es inútil todo intento de medir el valor. Al intercambio ni le precede ni le acompaña ningún proceso que implique tasar ni ponderar. Si un individuo atribuye el mismo valor a dos cosas, no tiene por qué intercambiar una cosa por otra. Si son distintamente valoradas, lo más que cabe afirmar es que una de ellas a, se valora en más (se prefiere a b). El valor y las valoraciones son expresiones intensivas. No puedan ser objeto de comprensión mental mediante números cardinales.

Sin embargo, se hallaba tan enraizada aquella errónea idea según la cual los valores eran medibles, al concertarse toda transacción económica; que incluso economistas clásicos y keynesianos incidieron en ese error. Ellos admitían la posibilidad de

capitalista de producción; se resistían a admitir que tales percances eran fruto exclusivo de los manejos de esos intervencionistas que pretenden rebajar la tasa del interés mediante la expansión crediticia. Los catedráticos de economía consideraban "poco científico" explicar la depresión como fenómeno provocado "exclusivamente" por hechos ocurridos en la esfera del dinero y del crédito. Hubo, incluso, quienes estudiaron la historia de los ciclos sin aludir a cuestiones monetarias. 


\section{El marco teórico de los errores y vacíos de la Teoría Monetaria Clásica y Keynesiana}

medir el valor, correspondiendo a la Economía explicar cómo se hace esa medición. Los economistas de segundo orden, sin dar mayor importancia al asunto, suponían que el dinero servía para medir el valor.

Valorar significa preferir $\mathbf{a}$ en vez de $\mathbf{b}$ y solo existe -lógica y psicológicamente- una sola forma de preferir. El mismo significado tiene el enamorado que prefiere una chica a las demás, y el consumidor que prefiere el pan a las golosinas. Preferir equivale a querer o desear a más que b. Por lo mismo que no cabe ponderar ni medir la atracción, la amistad, la simpatía o el placer estético, tampoco es posible calcular numéricamente el valor de los bienes. Cuando alguien intercambia dos kilos de mantequilla por una camisa, lo más que cabe decir de dicho acto es que el individuo en el momento de transar $y$ en las específicas circunstancias de aquel instante, prefiere una camisa a dos kilos de mantequilla. En cada acto de preferir, es diferente la intensidad psíquica del subjetivo sentimiento en que se basa tal acto. El ansia por alcanzar cierto fin puede ser mayor o menor; la vehemencia del deseo predetermina la cantidad de ese beneficio de orden psíquico; que la acción (cuando es idónea para provocar el efecto apetecido) proporciona al individuo que actúa. Sin embargo, las cantidades psíquicas, solo cabe sentirlas. Son de índole estrictamente personal y no es posible, por medios semánticos, expresar su intensidad ni informar a nadie acerca de su íntima condición.

No cabe arbitrar ninguna unidad de valor. Nunca tienen el mismo valor dos idénticas porciones de un cierto conjunto de bienes.

En el mercado aparecen los precios monetarios. El cálculo económico se efectúa en base a precios monetarios. Las diversas cantidades de bienes y servicios pueden ser tomadas en cuenta, al calcular; teniendo en cuenta las sumas dinerarias por las cuales han sido compradas y vendidas en el mercado. Es erróneo suponer que pueda calcular el individuo autárquico y aislado y el director de una República Autárquica, donde no existe un mercado para los factores de producción. Ninguna fórmula permite (partiendo del cálculo monetario, característico de la economía de mercado) calcular en un sistema económico donde no exista el mercado. 


\section{Pablo Rivas Santos}

\section{ERRORES Y VACÍOS DE LAS TEORÍAS MONETARIAS CLÁSICA Y KEY- NESIANA}

Si los economistas clásicos y keynesianos no se hubieran equivocado en materias referentes a los problemas monetarios, aferrándose después a sus errores, difícilmente podrían prevalecer todas esas nefastas políticas, inspiradas en populares teorías monetarias, que han desorganizado la política monetaria en casi todos los países.

Grave error fue suponer que el dinero era neutral. Tal supuesto indujo a creer que el nivel de precios sube y baja proporcionalmente al aumento o disminución de la cantidad de dinero en circulación. Se olvidaba que jamás ninguna variación de las existencias dinerarias puede; afectar a los precios al mismo tiempo y en la misma proporción. iLos cambios del poder adquisitivo del dinero son función de cambios en las relaciones entre compradores y vendedores! Para demostrar la procedencia de ese erróneo supuesto (según la cual la cantidad de dinero y los precios aumentan o disminuyen proporcionalmente) se adoptó (al abordar la teoría monetaria) una sistemática distinta a la sistemática que usa la moderna Economía para analizar los demás problemas económicos. En vez comenzar examinando actuaciones individuales, se comenzó examinando la economía de mercado en su conjunto. Ello obligaba a manejar conceptos como la cantidad total de dinero en la economía centralizada de mando; el volumen comercial (el equivalente monetario de todas las transacciones de bienes y servicios realizados en la economía centralizada de mando), la velocidad media de circulación del dinero; y el nivel general de precios. Tales arbitrariedades aparentemente hacían aceptable la teoría del nivel general de precios. Sin embargo, ese modo de razonar, supone pensar en típico círculo vicioso. La ecuación de intercambio presupone tal teoría del nivel general de precios que pretende demostrar. Tal ecuación es una expresión matemática de aquel -insostenible- supuesto según el cual existe uniforme proporcionalidad entre precios y variaciones de la cantidad de dinero ${ }^{2}$.

2 La ecuación del intercambio MV = PT fue formulada por Irving Fisher; siendo $\mathbf{M}$ la masa monetaria existente, $\mathbf{V}$ su velocidad de circulación, $\mathbf{P}$ el nivel general de precios y $\mathbf{T}$ el total volumen comercial. Tal planteamiento incurre en tautología, al afirmar que el precio pagado por todo lo comprado es igual al precio percibido por todo lo vendido; $y$, además, se basa en la supuesta existencia de identidad valorativa entre las partes en el acto de la compraventa, olvidando que el intercambio -ya sea directo o indirecto- exige que las partes valoren de modo diferente lo que dan y lo que reciben, pues, en otro caso, no habría operación posible. Además, presupone, lo que precisamente quiere probar, es decir, la existencia de un cierto nivel de precios, concepto también rechazable; pues lo que hay en el mercado son multiplicidad de bienes con sus respectivos precios que tenderán a subir, ceteris paribus, si aumenta el volumen monetario; pero, en ningún caso, lo harán uniformemente ni de modo simultáneo. Mises ataca la idea de la neutralidad del dinero, según la cual este no constituiría más que simple factor multipli- 


\section{El marco teórico de los errores y vacíos de la Teoría Monetaria Clásica y Keynesiana}

Al examinar la ecuación de intercambio, se supone que uno de sus elementos varía: -la cantidad total de dinero, el volumen comercial, la velocidad de circulación-, sin que nadie se pregunte cuál es la causa motivadora de tal variación. Esa variación no aparece, en la economía, por generación espontánea; lo que varía es la disposición personal de los individuos que actúan en la economía, siendo las múltiples actuaciones de tales individuos lo que provoca esa variación que registra la estructura de precios. Los economistas matemáticos ocultan esa oferta y demanda de dinero desatada por cada uno de los individuos participantes en la economía. En cambio, recurren al engañoso concepto de la velocidad de la circulación basado en ideas tomadas de la mecánica.

No interesa discutir si los economistas matemáticos tienen o no tienen razón cuando afirman que los servicios que presta el dinero radican, exclusivamente, en la circulación del dinero. Aun cuando esa afirmación fuera cierta, no por ello dejaría de ser ilógico basar en tales servicios; la capacidad adquisitiva -el precio- del dinero. Los servicios que prestan al hombre el agua, la cerveza o el té no determinan los precios que el mercado paga por tales bienes. Dichos servicios nos hacen comprender por qué la gente, una vez que se da cuenta de las propiedades de aquellos bienes, demandan, en específicos casos, determinada cantidades de las mismas. Es la demanda, no el valor de uso objetivo, lo que determina los precios.

Tratándose del dinero, la teoría económica debe abordar problemas de mayor amplitud que aquellos problemas que analiza al ocuparse de los demás bienes. Compete a la psicología y fisiología, explicar por qué la gente desea esos demás bienes contratados en el mercado. Sin embargo, tal incógnita, cuando se trata del dinero, sí compete a la teoría económica despejarla. La teoría económica debe indicarnos qué ventajas pretende obtener el hombre, de la tenencia de dinero. No son tales ventajas las únicas circunstancias que determinan el poder adquisitivo del dinero. El deseo de disfrutar esas ventajas es, únicamente, uno de los factores que desata la demanda de dinero. Y es solo tal demanda, o sea, un factor subjetivo cuya intensidad exclusivamente depende de jui-

cador o divisor de los precios, en caso de aumento o reducción de su cuantía. Cualquier aumento de las existencias dinerarias influye sobre los precios, pero siempre en forma diferente y en momentos distintos, en razón a que, en caso de inflación, los nuevos medios de pago jamás engrosan, de golpe y en la misma cantidad, las tesorerías de todas las personas actuantes, enriqueciendo, primero, a ciertos grupos que, con sus compras, hacen subir específicos precios; y el alza solo paulatinamente va afectando a los demás sectores de! mercado. Se trata de un terremoto, que trastoca todos los precios. Y, en caso de reducción de las disponibilidades dinerarias, sucede lo mismo, solo que al revés. 


\section{Pablo Rivas Santos}

cios valorativos (nunca de hechos objetivos, ni de capacidad alguna para provocar efectos determinados) lo que da lugar a los tipos de intercambio que registra el mercado.

El defecto que adolece la ecuación de intercambio y los conceptos manejados en tal ecuación consiste en que contempla los fenómenos de mercado de un modo totalitario. La obsesión por la economía centralizada de mando lo confunde todo. Cuando aparece tal economía, se desvanecen los precios, el mercado y el dinero. En el mercado solo operan individuos o asociaciones de individuos. Son los propios intereses personales los que inducen a tales individuos a actuar, jamás aquellos hipotéticos intereses de la economía en su conjunto. Para que conceptos tales como el volumen comercial o la velocidad circulatoria tengan sentido, es necesario retrotraerlos a específicas actuaciones individuales. Lo que no es permisible es recurrir a esos mismos conceptos para explicar aquellas actuaciones personales que los originan. El primer problema que la economía de mando debe plantearse ante una variación de la total cantidad de dinero disponible en el mercado; es el problema de cómo dicho cambio puede afectar la conducta de los diversos individuos actuantes. La economía moderna no pretende averiguar cuánto vale el acero o el pan, sino cuánto vale cierta cantidad de acero o de pan para específico individuo que actúa en determinado tiempo y lugar. Por tanto, del mismo modo, debemos proceder cuando se trata del dinero. La ecuación de intercambio pugna con los principios básicos que informan el pensamiento económico. Implica recaer en aquellos ya superados modos de pensar, típicos de épocas primitivas, en que la gente no comprendía los fenómenos económicos precisamente porque partían de conceptos totalitarios. Estéril es tal sistemática, por lo mismo que eran inútiles los arcaicos razonamientos que pretendían averiguar el valor general del acero o del pan.

La teoría del dinero es un capítulo más de la teoría económica. Por tanto, el tema debe abordarse idénticamente como se analizan todos los demás problemas de la teoría económica.

\section{LA FICCIÓN DE LAS POLÍTICAS CONTRACÍCLICAS}

Las teorías heterodoxas de los intervencionistas afirman que las crisis económicas son fruto inevitable de la propia economía de mercado, provocándolas el vicioso funcionamiento de la economía de mercado. Los intervencionistas creen que cabe evitar las

crisis cíclicas si el gobierno interviene oportuna y convenientemente. El gobierno puede 


\section{El marco teórico de los errores y vacíos de la Teoría Monetaria Clásica y Keynesiana}

imponer la estabilidad económica. Nada cabría objetar a tales intervencionistas si, para evitar las depresiones, lo que pidiera al gobierno fuera eliminar la expansión crediticia. Sin embargo, el intervencionista, de antemano rechaza tal solución. Antes al contrario, aspira a intensificar la expansión crediticia, pretendiendo evitar la crisis cíclicas mediante políticas contracíclicas.

El gobierno se aparece a tales economistas como un poderoso situado fuera de la órbita de los negocios humanos, completamente independiente de los agentes; con poder para influir en la vida y aspiraciones de los agentes. Imaginan que el gobierno dispone de fondos propios, ilimitados, que ni proceden de los gobernados ni nada tienen que ver con los gobernados. Tales fondos, se supone, el gobierno puede libremente destinarlos a cuanto más crean conveniente. Corresponde a los expertos dictaminar acerca de cuáles sean las inversiones a que dichas sumas deban dedicarse.

Las dos políticas contracíclicas consisten en construir obras públicas e invertir grandes sumas de dinero en empresas estatales. Sin embargo, tales fórmulas no son tan originales como suponen sus partidarios. La gente -desde hace tiempo- en cuanto surgía la temida crisis, reclamaban el inicio de ambiciosos proyectos que crearan trabajo y detuvieran la caída de precios. La propuesta, a primera vista, parece plausible; pero el verdadero problema consiste en cómo financiar tales obras. Si el gobierno incrementa los impuestos o lanza empréstitos, en nada aumenta el gasto conjunto total; pues reduce la capacidad de consumo e inversión privada en igual medida que incrementa la propia. En cambio, de recurrir, a la inflación, en vez de mejorar, empeora las cosas. Quizá consiga, así, el gobernante diferir por algún tiempo el estallido de la crisis. Sin embargo, cuando llegue el inevitable final, la depresión será tanto más violenta cuanto por más tiempo el gobierno haya venido aplazándola. Los expertos del dirigismo no acaban de percatarse del problema subyacente. Lo fundamental para ellos es tener bien preparados "los correspondientes planes de inversión pública y articulados los grandes proyectos que habrán de ponerse en marcha al primer signo de peligro". Dicen: este es "el método más acertado, cuya adopción recomendamos a todos los países". Sin embargo, el problema no consiste en elaborar proyectos, sino en hallar los medios necesarios para su ejecución. Algunos dirigistas afirman que cabía arbitrar los correspondientes factores de producción sobre la base de frenar las inversiones públicas durante la euforia; tales retenidos fondos serían lanzados al mercado al sobrevenir la crisis. 


\section{Pablo Rivas Santos}

No está mal restringir el gasto estatal. Sin embargo, con ello no se provee al tesoro público los recursos que más tarde necesitará para efectuar las deseadas inversiones. La persona individual puede proceder así; le es dable crear reservas cuando tiene mayores ingresos y disponer de lo ahorrado en el momento en que sus ganancias disminuyen. Pero la cosa es distinta cuando se trata de un país. Al tesoro público le cabe retener una parte de los abundantes recursos fiscales resultantes de la euforia alcista. En tanto tales recursos queden sustraídos de la circulación; se estará practicando una política deflacionaria y contracíclica que puede frenar la euforia generada por el nuevo dinero puesto en circulación. Si tales recursos son de nuevo lanzados al mercado se modificará con ello la relación monetaria, reduciéndose el poder adquisitivo del dinero. En ningún caso dichos recursos pueden engendrar los factores de producción exigidos por las proyectadas obras públicas.

\section{CONCLUSIONES}

Los intervencionistas coinciden en el error de ignorar que el capital disponible es escaso. En su opinión, la crisis, aparece porque de pronto se apodera de la gente una misteriosa aversión a invertir y consumir. Cuando lo que interesa de verdad es incrementar la producción y restringir el consumo, para aumentar así el capital disponible, los intervencionistas quisieran ampliar al mismo tiempo el consumo y la inversión. Estos exigen que el gobierno efectúe empresas que no son rentables, porque los correspondientes factores de producción deben sustraerse a otras producciones que permiten atender necesidades que los consumidores consideran más urgentes que aquéllas que la acción estatal cubrirá. No advierten que las grandes obras públicas que recomiendan no sirven sino para empeorar las cosas, al venir a incrementar la escasez de capital.

Cabría teóricamente imaginar un distinto uso de esas reservas acumuladas durante el período de la euforia alcista. El tesoro público podría destinar tales reservas a la compra de todos aquellos factores de producción y artículos de consumo que más tarde, al sobrevenir la depresión, habrá de invertir en las mencionadas obras públicas proyectadas y en atender las necesidades de quienes en ellas trabajan. Sin embargo, las autoridades si así procediesen, impulsarían la euforia alcista, acelerando el estallido de la crisis y agravando la crisis ${ }^{3}$.

3 Los intervencionistas, al referirse a las políticas contracíclicas, invocan los supuestos éxitos que tal política tuvo en los períodos 1950-1970, 1986-1987. Cierto es que los gobiernos duplicaron sus gastos e inversiones. Sin embargo, ello fue efecto de la prosperidad que el país disfrutada gracias a las reservas y coyuntura económica. Incrementar enormemente sus exportaciones y, además, se vieron libres de la competencia en el mercado internacional de minerales. Las exportaciones aumentaron, por esta razón (en miles de toneladas). 


\section{El marco teórico de los errores y vacíos de la Teoría Monetaria Clásica y Keynesiana}

Con tantos discursos de las políticas contracíclicas se pretende confundir a la gente e impedir a la gente advertir cuál es la verdadera causa provocadora de las fluctuaciones cíclicas. Actualmente, las autoridades se hallan comprometidas, a una política de intereses bajos, de expansión crediticia y de inflación. Y luego, cuando afloren las inevitables consecuencias de tales manipulaciones, el gobierno provocará nuevas y mayores inflaciones.

\section{BIBLIOGRAFÍA}

Friedman, Milton (1960). Políticas monetarias de estabilización. Argentina: Editorial Ateneo, pp. 310-350.

Friedman, Milton (1980). Moneda y desarrollo. España: Editorial Ateneo, pp. 123152.

Friedman, Milton (1987). Nueva reformulación de la teoría cuantitativa del dinero. España. Editorial Continental. Primera edición 1971, pp. 123-152.

Lucas, Robert (1987). Teoría de los ciclos económicos. España: Alianza Editorial, pp. 113-152.

Mundell, Robert A. (1971). Teoría monetaria. España: Ediciones Amorrurtu Editores, pp. 323-430.

Von Hayek Friedrich (1978). Desnacionalización del dinero. España: Editorial Continental, pp. 143-187.

Von Mises Ludwig (1966). Teoría de la moneda y el crédito. España: Fundación Ignacio Villalonga, pp. 239-452.

Von Mises Ludwig (1980). Acción humana. España. Fundación Ignacio Villalonga, pp. 180332 